\title{
P2X7 blockade inhibits the growth of breast cancer in 4T1 breast cancer-bearing mice by NLRP3/caspase 1 pathway
}

Xiaolan Yu ${ }^{1}$, Xuejiao Chen ${ }^{1}$, Xiaoping Tang 2 , Yong $\mathrm{CaO}^{2}$, Li Tang 2 , Tao He 3 , Yong Liu ${ }^{4}$

\author{
${ }^{1}$ Department of Obstetrics and Gynecology, Affiliated TCM Hospital of Southwest \\ Medical University, Sichuan, China \\ ${ }^{2}$ Medicine Experimental Center, The Affiliated Hospital of Southwest Medical \\ University, Sichuan, China \\ ${ }^{3}$ Cancer Research Institute of SouthWest Medical University, Sichuan, China \\ ${ }^{4}$ Department of Pathology, Affiliated Hospital of Southwest Medical University, \\ Sichuan, China
}

Submitted: 26 March 2020

Accepted: 21 June 2020

Arch Med Sci

DOI: https://doi.org/10.5114/aoms.2020.99013

Copyright $\odot 2020$ Termedia \& Banach

\section{Abstract}

Introduction: The role of $\mathrm{P} 2 \mathrm{X} 7$ in the progression of breast cancer remains unclear; hence, it is necessary to investigate whether the P2X7/NLRP3/ caspase 1 signalling pathway is associated with the development of breast cancer.

Material and methods: 4T1 breast cancer-bearing mice models were developed for P2X7 agonists BzATP and antagonists BBG. The weight of breast cancer tissue among groups was calculated and compared. The cancer tissue was observed by haematoxylin and eosin (HE) staining, and the expression of P2X7, NLRP3, and caspase 1 was examined by immunofluorescence and western blot. Results: The tumour weight and the medullary lymphocytes in the BzATP group were significantly higher than those of the sham and control groups, but the tumour weight and the medullary lymphocytes in the BBG group were significantly lower than those of the sham, control, and BzATP groups. The number of positive P2X7 in the BzATP group was significantly higher than that of other groups, but BBG significantly reduced the number of P2X7. The relative expression level of P2X7 in the BzATP group was significantly higher than that of other groups, but the relative expression level of $\mathrm{P} 2 \mathrm{X7}$ in the BBG group was significantly lower than that of other breast cancer-bearing groups.

Conclusions: The blocking of $\mathrm{P} 2 \times 7$ can inhibit the growth of breast cancer in $4 \mathrm{~T} 1$ breast cancer-bearing mice via NLRP3/caspase 1 pathway. Future studies are needed to elucidate the underlying mechanism.

Key words: breast cancer, P2X7, NLRP3, caspase 1.

\author{
Corresponding author: \\ Yong Liu \\ Department of Pathology \\ The Affiliated Hospital \\ Southwest Medical \\ University \\ Luzhou, Sichuan, China \\ E-mail: yongliu2001@sohu. \\ com
}

\section{Introduction}

Breast cancer is the most common cancer in women and a commonly seen cause of death among women worldwide. In China, it has been reported that deaths due to breast cancer have accounted for $6.9 \%$ of all cancer deaths [1, 2]. It has been reported that breast cancer is associated with increased health burden, reduced life quality, and decreased life expectancy $[3,4]$. The early detection and diagnosis of breast cancer is es- 
sential to the prognosis of the patients. Currently, despite the significant benefits of using conventional chemotherapy and monoclonal antibodies such as anti-PD-L1 antibody in the treatment of breast cancer patients, the development of effective treatments for breast cancer remains a huge challenge for health care providers [5-7].

The tumour microenvironment (TME) plays a very important role in the occurrence and development of breast cancers [8,9]. Recent studies [10-12] have shown that extracellular ATP is likely to be an important invasive factor in TME. The ATP-gated receptor P2X7 is expressed in multiple malignant tumours, including breast cancers [13]. Furthermore, it has been reported that P2X7R is the key mediator of ATP to promote the invasion of cancer growths [14]. P2X7R is the most unique subtype of the purine $\mathrm{P} 2$ receptor family, and so research on the structure, physiological characteristics, and functions of P2X7R has progressed rapidly in recent years. Multiple studies [15-17] have shown that P2X7R plays an important role in the proliferation and apoptosis of breast cancer cells and has different expression levels in normal breast tissue, precancerous lesions, and breast cancer tissues. P2X7R is a potent stimulant of inflammation and immunity and a promoter of cancer cell growth [18]. This makes P2X7R an appealing target for anti-inflammatory and anti-cancer therapy.

It has been reported [19] that the P2X7/NLRP3/ caspase 1 pathways are closely linked to Alzheimer's disease $(A D)$ via neuroinflammation. And previous studies [20-22] have reported that $\mathrm{P} 2 \mathrm{X} 7$ can activate NOD-like receptor pyrin-domain-containing 3 (NLRP3), thereby activating inflammato- ry factors and inducing inflammation, leading to a TME that is more favourable to the growth of cancers. Based on a literature review, to date no reports on the role of P2X7-NLRP3 pathway in the breast cancer have been found.

Therefore, we attempted to investigate the hypothesis that the $\mathrm{P} 2 \mathrm{X} 7 / \mathrm{NLRP} 3 /$ caspase 1 signalling pathway may be relevant in the occurrence and growth of breast cancer.

\section{Material and methods}

\section{Ethical considerations}

All the animal experiment protocols were certified and approved by the Animal Care and Use Committee of our hospital (20170912) and complied with the guidelines of Animal Research: Reporting of In Vivo Experiments (ARRIVE). Our study was approved by the Ethics Committee of our Hospital (20170912).

\section{Animals}

Female Balb/C mice with an age of 5-6 weeks were used in this study (Animal Centre of Chinese Academy of Sciences, China). During the period of the experiment, all animals were housed three per cage and in a temperature-controlled $\left(23-26^{\circ} \mathrm{C}\right)$ environment with a 12 -hour light/day cycle and free access to food and water. For the development of the breast cancer-bearing model, the related mice were injected orthotopically with $1 \times 10^{4} 4 \mathrm{~T} 1$ murine breast cancer cells into one right thoracic mammary gland [23]. Thirty days after injection the mice were used for further intervention. Subsequently, all the animals were eu-

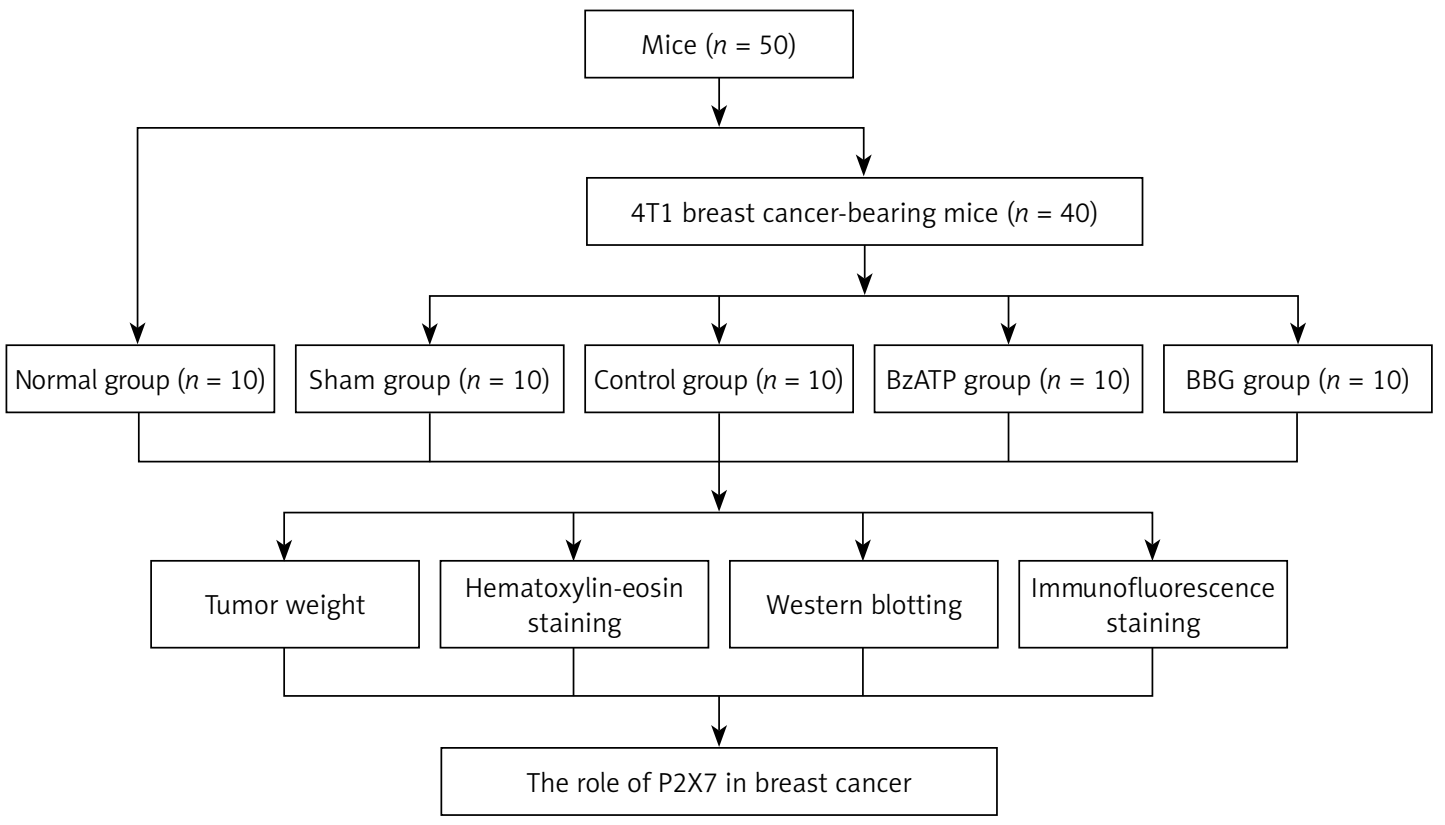

Figure 1. The flow chart of study design. BzATP is a P2X7 agonist, and BBG is a P2X7 antagonist 
thanised by i.p. injection of ketamine and xylazine (100 and $10 \mathrm{mg} / \mathrm{kg}$, respectively).

\section{Experimental design}

The experimental design is shown in Figure 1. Fifty Balb/C mice were randomly distributed to the normal group, sham group, control group, BzATP (P2X7 agonist) group, and BBG (P2X7 antagonist) group, with 10 mice in each group. The sham, control, BzATP, and BBG groups all comprised $4 \mathrm{~T} 1$ breast cancer-bearing mice; the mice in the sham group received no intervention, the mice in the control group received $2 \mathrm{ml}$ demineralised water injected intraperitoneally, and mice in the BzATP and BBG groups received BzATP and BBG intervention, respectively. BzATP (soluble in demineralised water, $50 \mu \mathrm{g} / \mathrm{kg}, 2 \mathrm{ml}$, SigmaAldrich, Shanghai, China) was intraperitoneally administered $1 \mathrm{~h}$ after 4T1 murine breast cancer cell injections, and BBG (soluble in demineralised water, $30 \mathrm{mg} / \mathrm{kg}, 2 \mathrm{ml}$, Sigma-Aldrich, Shanghai, China) was intraperitoneally administered [24]. All of the P2X7 agonist and antagonist were used in one dose, and animals were sacrificed seven days after drug injection,

\section{Tumour weight calculation}

The food was inaccessible to mice $6 \mathrm{~h}$ before sacrifice. The mice were sacrificed by the method of cervical dislocation, then the tumour was carefully peeled off with delicate equipment in sterile conditions, and filter paper was used to absorb the related blood around the tumour whenever necessary. Finally, the tumour was weighed with an electric balance ( $\mathrm{S3080}$, Xinghe, Nanjing), and the related weight was recorded accordingly.

\section{Haematoxylin and eosin (HE) staining}

The cancer tissues were weighed and dehydrated, and then embedded in paraffin. Furthermore, the transverse section of collected tissues was discreetly sliced for HE staining, as previously reported [25]. The specimens then were photographed and analysed with a microscope (Olympus S100, Japan) at 40x magnification. For data analysis, we calculated and compared the number of lymphocytes at up to 10 pictures for each group.

\section{Western blotting}

The frozen tissue samples were lysed mechanically in cell lysis buffer for total protein extraction. Protein extraction kits (P0033, Beyotime, China) were used. The lysates were centrifuged at 12,000 rpm for $15 \mathrm{~min}$ at $4^{\circ} \mathrm{C}$, and the concentration was measured by the bicinchoninic acid (BCA) method. The samples were further separated using related
SDS-PAGE and were electro-transferred onto nitrocellulose membrane. Then, the membranes were blocked with $5 \%$ non-fat milk for $1 \mathrm{~h}$ at room temperature and were further incubated with primary antibodies at $4^{\circ} \mathrm{C}$. In this present study, $\beta$-tublin (ab179513, Abcam, USA) was used as a loading control. The membranes were then washed three times for $5 \mathrm{~min}$ each in TBS + 0.1\%Tween 20 and were then incubated in the appropriate secondary antibodies for $2 \mathrm{~h}$ at room temperature. Finally, the protein bands were visualised with the method of enhanced chemiluminescence, and the relative expression level of detected proteins was processed and analysed with Image J software. The antibodies used in this study included the following: rabbit anti-P2X7 (ab48871, Abcam, USA), rabbit anti-NLRP3 (ab214185, Abcam, USA), and rabbit anti-caspase 1 (ab238979, Abcam, USA).

\section{Immunofluorescence staining}

The immunofluorescence staining was conducted in concordance with the related guidelines [26]. Generally, the tumour sections were incubated with primary antibodies overnight at $4^{\circ} \mathrm{C}$ and were further covered with secondary antibodies (Jackson Immunoresearch, West Grove, PA) for $2 \mathrm{~h}$ at room temperature accordingly. We observed the subcellular localisation of proteins with a confocal laser scanning microscope (Olympus Z600, Japan).

\section{Statistical analysis}

We used SPSS22.0 software (SPSS Chicago, USA) for the statistical processing and analysis. All the collected data were expressed as mean \pm standard deviation (SD) in the present study. We compared the differences between groups with oneway ANOVA method followed by a post hoc test (LSD). $P<0.05$ was considered as being statistically significant in this study.

\section{Results}

\section{Tumour weights}

As can be seen in Figure 2, the tumour weight in the BzATP group was significantly higher than that of the sham and control groups, but the tumour weight in the BBG group was significantly lower than that of the sham, control, and BzATP groups.

\section{HE staining analysis}

As can be seen in Figure 3, for the normal group, the structure of breast tissue was complete and relatively independent. Meanwhile, for the other breast cancer group, the hyperplasia of mammary glands was obvious, and the structure was not clear. Furthermore, the medullary lymphocytes in the BzATP group were significantly higher 

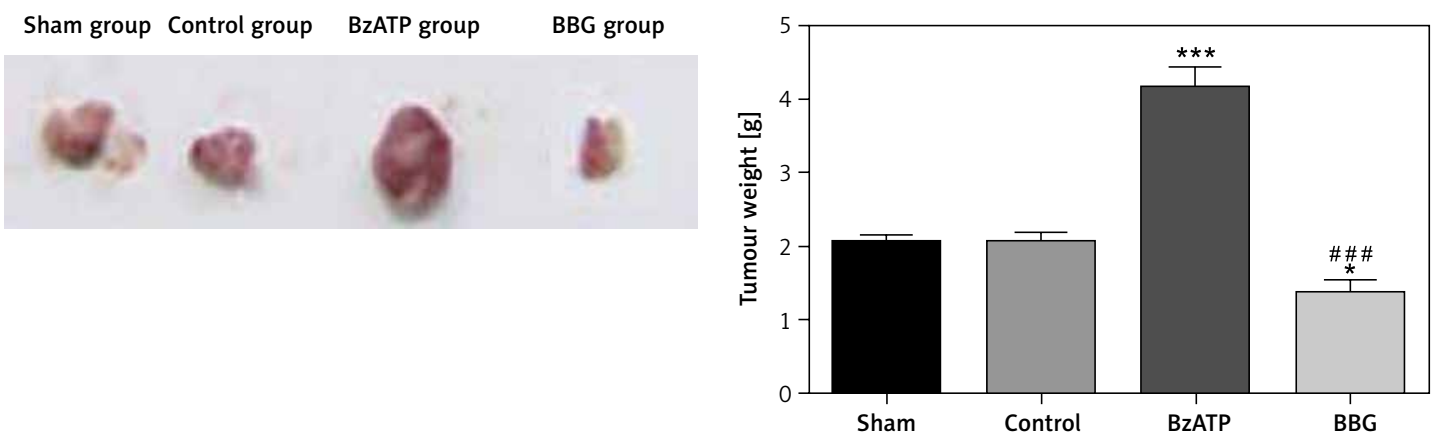

Figure 2. Tumour outlook and tumour weight comparison among groups

${ }^{* * *} p<0.001$ vs. control, ${ }^{*} p<0.05$ vs. control, ${ }^{\# \# *} p<0.001$ vs. BzATP.
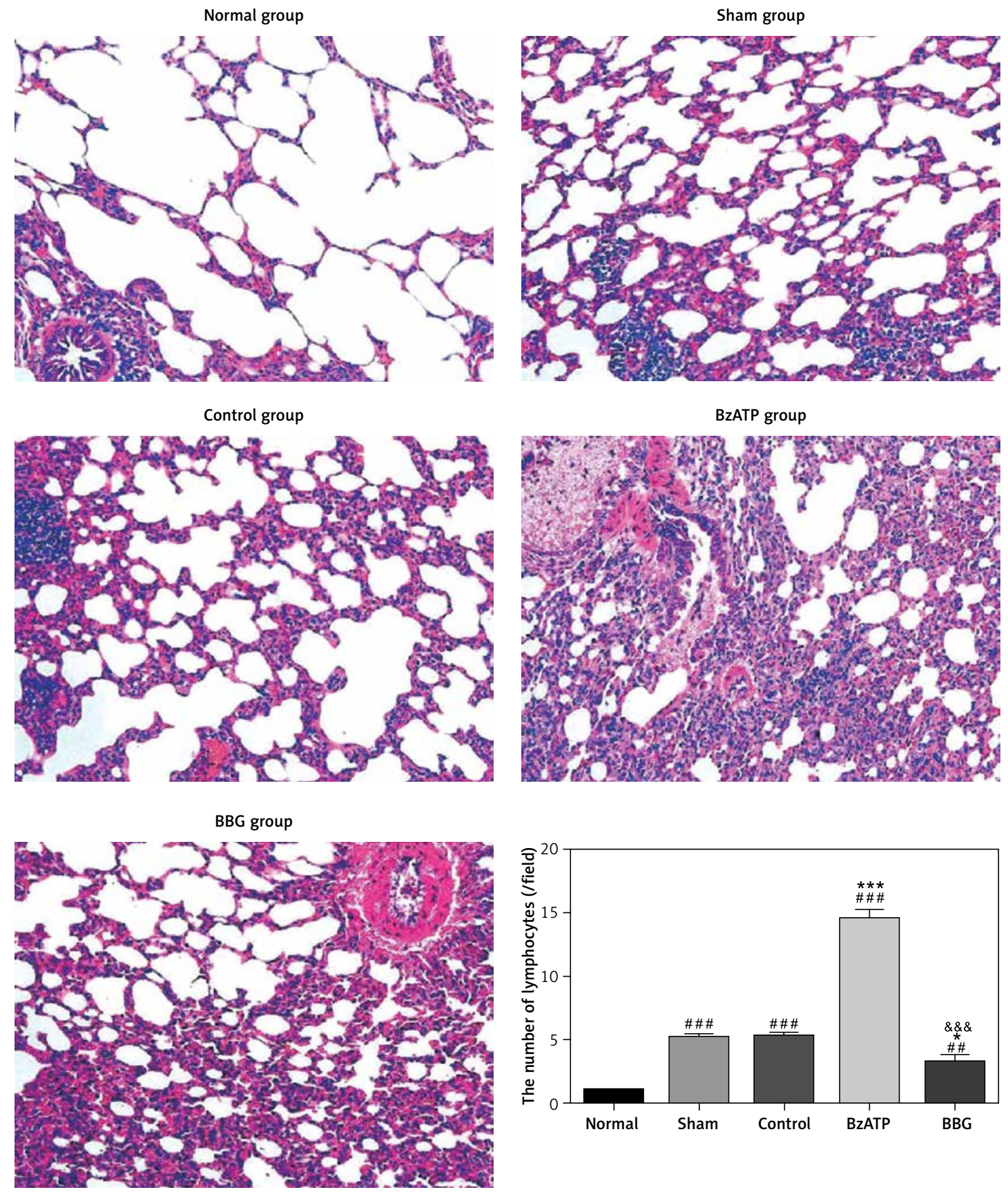

Figure 3. HE staining analysis among groups

${ }^{\# \# \# p} p<0.001$ vs. normal, ${ }^{* * *} p<0.001$ vs. control, ${ }^{*} p<0.05$ vs. control, ${ }^{\& \& \&} p<0.001$ vs. BzATP. 
than those of the other groups, but the medullary lymphocytes in the BBG group were significantly lower than those of the sham, control, and BzATP groups.

\section{Immunofluorescence staining analysis}

As can be seen in Figure 4, the immunofluorescence staining indicated that the number of positive P2X7 was significantly increased for breast cancer-bearing groups, and the number of positive P2X7 in the BzATP group was significantly higher than that of the other groups, but the BBG significantly reduced the number of $\mathrm{P} 2 \mathrm{X} 7$.

\section{Western blot analysis of expression of $\mathrm{P} 2 \mathrm{X} 7$}

As can be seen in Figure $5 \mathrm{~A}$, the relative expression level of $\mathrm{P} 2 \mathrm{X} 7$ was significantly increased for breast cancer-bearing groups, and the relative expression level of P2X7 in the BzATP group was significantly higher than that of other groups, yet the relative expression level of $P 2 X 7$ in the $B B G$ group was significantly lower than that of other breast cancer-bearing groups.

\section{Western blot analysis of the expression of NLRP3 and caspase 1}

As can be seen in Figures $5 \mathrm{~B}$ and $\mathrm{C}$, the relative expression level of NLRP3 and caspase 1 were significantly increased for breast cancer-bearing groups, and the relative expression levels of NLRP3 and caspase 1 in the BzATP group were signifi- cantly higher than those of other groups, but the relative expression levels of NLRP3 and caspase 1 in the BBG group were significantly lower than those of other breast cancer-bearing groups.

\section{Discussion}

With the progression of research, it has been found that abnormal expression of $\mathrm{P} 2 \mathrm{X} 7$ is related to the occurrence and development of various cancers, and it may even become a new target for cancer treatment and prognosis [14, 27]. Therefore, it is necessary to conduct more related studies to elucidate the mechanism of P2X7 on the development and treatment of breast cancer. The results of the present study confirm that P2X7 plays an important role in the development of breast cancer, downregulating $\mathrm{P} 2 \times 7$ can inhibit the growth of breast cancer, and that the P2X7/ NLRP3/caspase-1 signalling pathway is closely involved in this process.

The $\mathrm{P} 2 \mathrm{X} 7$ receptor is a member of the $\mathrm{P} 2 \mathrm{X}$ family, with unique structure and function. Its biological functions include participating in cell signal transduction, cytokine secretion, and mediating cell survival and growth [28, 29]. The production of high concentrations of ATP after tissue damage activates the $P 2 X 7$, causing the transient opening of cell membrane pathways and leading to an influx of calcium ions. Subsequently it catalyses the hydrolysis of phosphatidylcholine to produce a second messenger phospholipid (PA) [30]. It further activates the Src kinase family (SFKs), hydrolyses protein precursors, and forms ligands, which
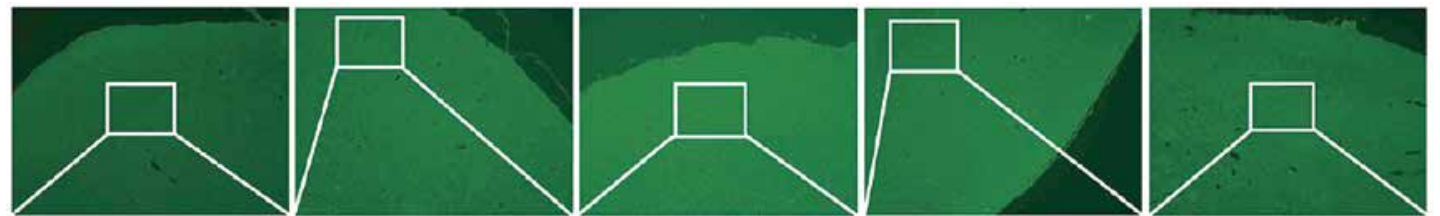
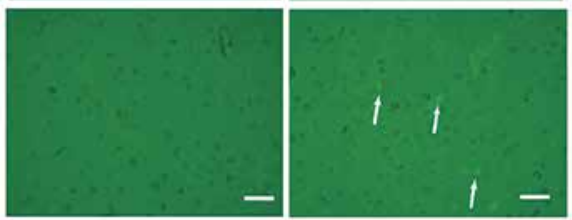

Normal group
Sham group

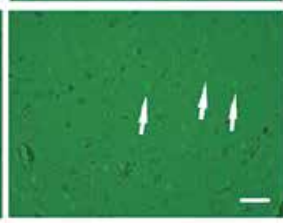

Control group

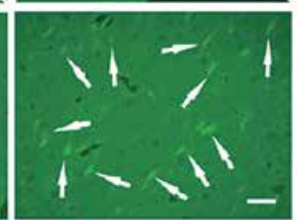

BzATP group

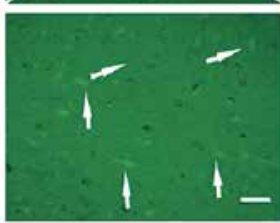

BBG group

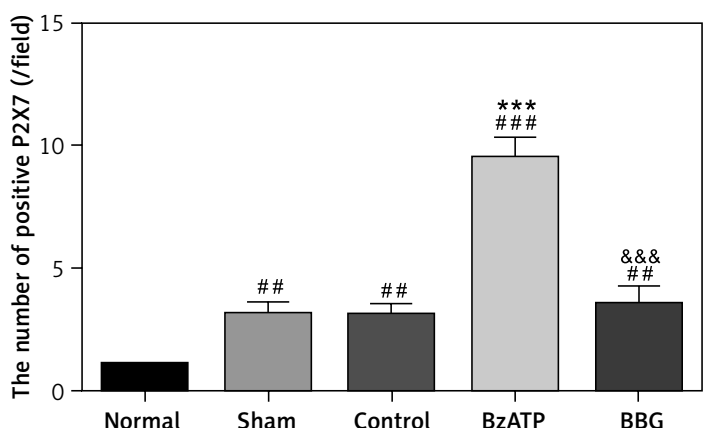

Figure 4. Immunofluorescence staining analysis among the groups

${ }_{\# \# P}<0.001$ vs. normal, ${ }^{\#} p<0.01$ vs. normal,

$\& \& \& p<0.001$ vs. BzATP. 
A
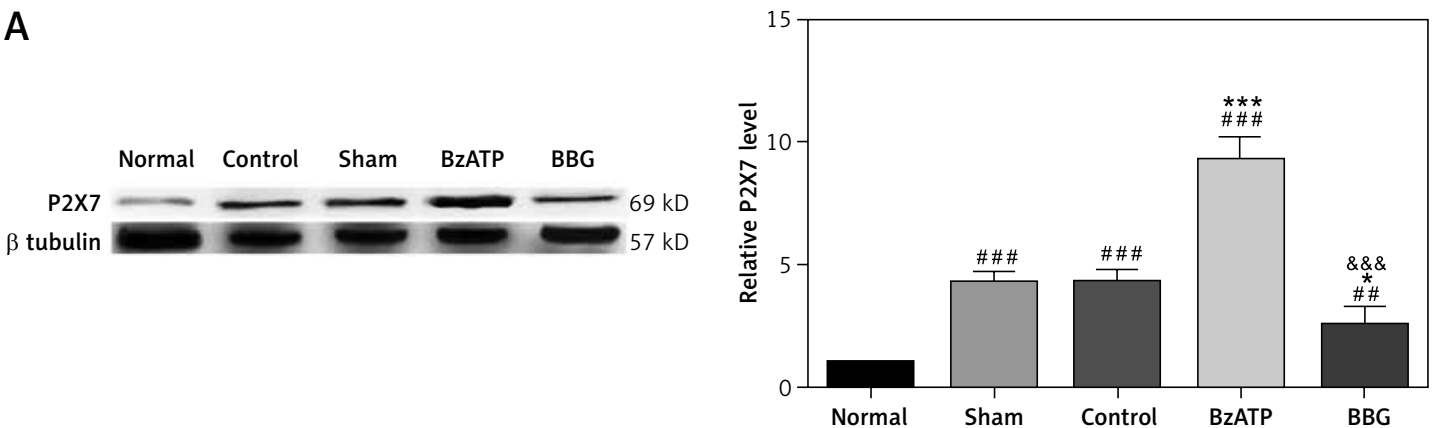

B
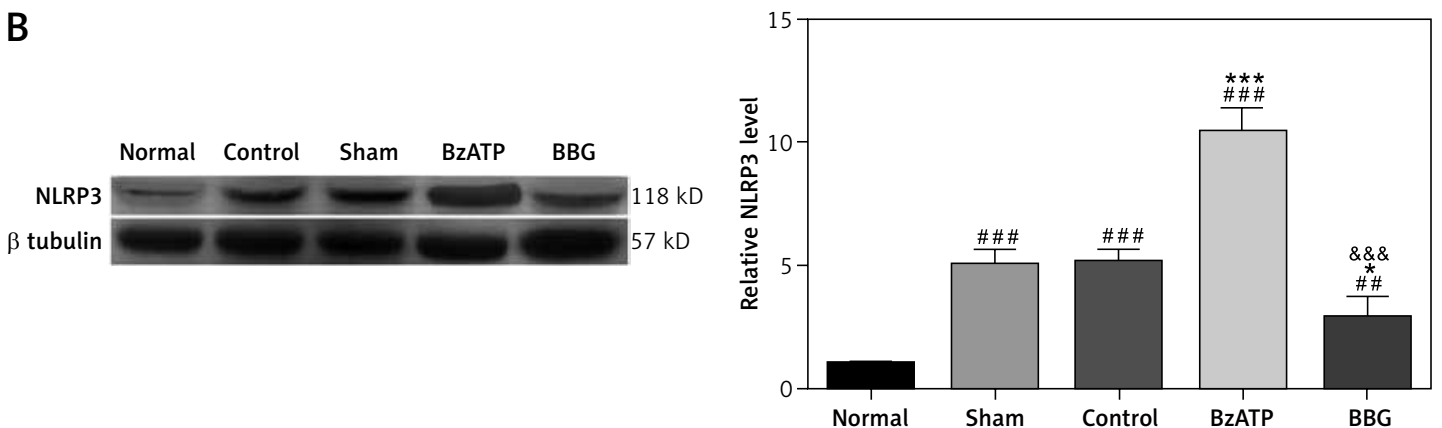

C
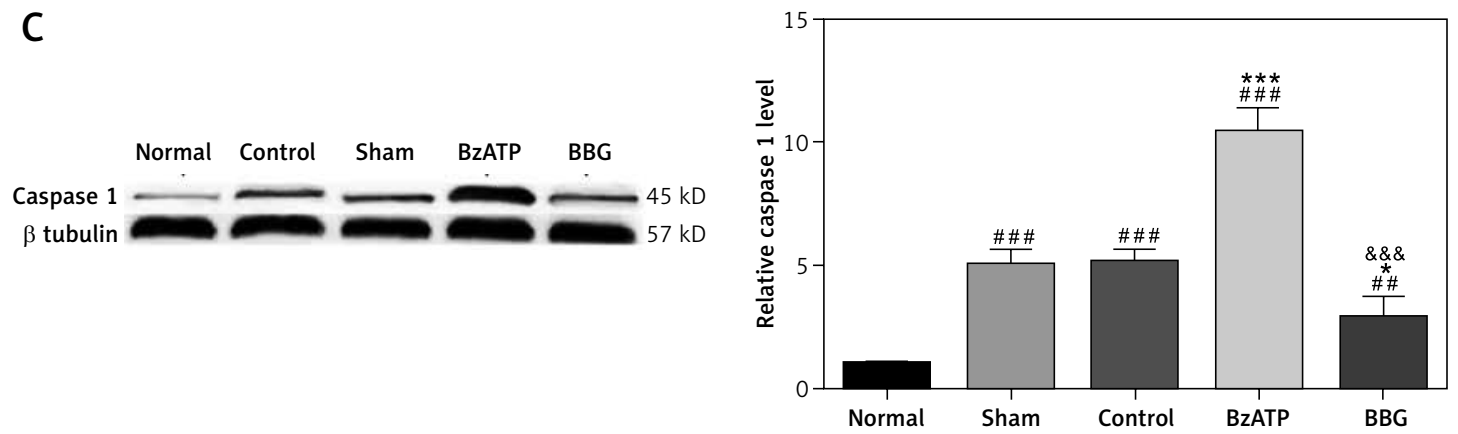

Figure 5. Western blotting analysis on the P2X7, NLRP3, and caspase 1 expression levels among groups

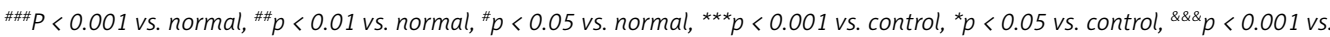
BzATP, ${ }^{\& \&} p<0.01$ vs. BzATP.

then bind to VEGFR, which in turn promotes the growth of tissues [31]. Also, the binding of ATP to the $\mathrm{P} 2 \mathrm{X} 7$ receptor induces the formation of membrane pores of lysed cells, which can lead to cellular $\mathrm{Ca}^{2+}$-independent cell necrosis [24, 32]. On the other hand, continuous ATP stimulation activates $\mathrm{P} 2 \mathrm{X} 7$ to trigger excessive $\mathrm{Ca}^{2+}$ to enter cells and induce apoptosis [33]. Therefore, P2X7 is closely associated with the development of cancers.

Breast cancer is a malignant cancer with high incidence. Previous studies [12, 34] found that the expression of P2X7 in normal, mildly proliferative breast epithelium is almost absent, while $\mathrm{P} 2 \mathrm{X} 7$ receptors are overexpressed in the ductal or lobular carcinoma. Also, it has been reported that the ATP concentration in the tumour stroma is significantly higher than that of normal tissues [35]. Therefore, P2X7 is likely to have a pro-proliferative effect in the development of breast cancer. At the same time, the expression of $\mathrm{P} 2 \mathrm{X} 7$ and oestrogen receptor $(\mathrm{OR})$ in breast cancer tissues has a posi- tive correlation [17]. The expression of P2X7R is abnormal in human MDA-MB-435s breast cancer cells and A549 lung adenocarcinoma cells, and the application of the P2X7R inhibitor KN-62 can block ATP-induced tumour cell migration and invasion [36], which is consistent with our findings.

Inflammatory response is a key factor in tumourigenesis and development, and its secreted multiple inflammatory factors can mediate the emergence of the tumour microenvironment. Also, it plays an important role in initiating, maintaining, and promoting tumour growth [37]. Of these, NLRP3 influences the pathogenesis of cancer by modulating innate and adaptive immune responses, cell death, proliferation, and gut microbiota [38]. NLRP3 can be assembled with the adaptor protein ASC and caspase 1 to form a multi-protein complex called an inflammasome. The assembly of inflammasome leads to the activation of caspase 1 and the maturation and secretion of pro-inflammatory cytokines such as IL-18, 
thus exerting a variety of immunological effects $[39,40]$. The results of our studies confirm that NLRP3 plays an important role in the progression of breast cancers.

Several limitations in this study must be considered. Firstly, we only performed a primary investigation on the role of $\mathrm{P} 2 \mathrm{X} 7 / \mathrm{NLRP} 3 /$ caspase 1 pathway in breast cancers. The ERK and JNK pathways should be further investigated in more detail because they are also a part of this signalling pathway [41]. The underlying mechanisms of how these synergistic effects work remain unclear, which also warrants further investigation. Secondly, activation of P2X7 leads to calcium mobilisation, which may be the mechanism link of P2X7 response to cells. We did not measure intracellular calcium mobilisation in this study; future studies focusing on calcium mobilisation measurement will help us confirm the acting mechanism of P2X7 in breast cancer. Thirdly, intro studies should be conducted to confirm the role of P2X7 in breast cancers; we will verify this in our future studies.

In conclusion, $\mathrm{P} 2 \mathrm{X} 7$ plays an important role in the development of breast cancer; blocking P $2 \times 7$ can inhibit the growth of breast cancer, and it is associated with NLRP3/caspase 1 pathway. It is worth noting that $\mathrm{P} 2 \mathrm{X} 7$ activation involves the activation of multiple different intracellular effector pathways, resulting in complex biological effects, and its application to clinical treatment has a long way to go - further explorations on the mechanisms of the antitumour effect of P2X7 are needed.

\section{Acknowledgments}

This research received grants from the following projects:

1. The mutual fund of Science \& Technology Department of Sichuan province (2015LZCYD-S01(12/15),

2. The project of education department of Sichuan province (15TD0020),

3. The project of Science \& Technology Department of Sichuan province (2013JY0076),

4. The project of Science \& Technology Department of Luzhou City (2013LZLY-J12).

Xiaolan Yu and Xuejiao Chen - equal contributor.

\section{Conflict of interest}

The authors declare no conflict of interest.

\section{References}

1. Liang D, Li D, Liu J, et al. Trends and patterns of cancer mortality in North China (Hebei Province), 1973-2013. Sci Rep 2018; 8: 311.

2. Fan L, Strasser-Weippl K, Li JJ, et al. Breast cancer in China. Lancet Oncol 2014; 15: e279-89.
3. Momenimovahed Z, Salehiniya H. Epidemiological characteristics of and risk factors for breast cancer in the world. Breast Cancer 2019; 11: 151-64.

4. Coughlin SS. Epidemiology of breast cancer in women. Adv Exp Med Biol 2019; 1152: 9-29.

5. Garcia-Aranda M, Redondo M. Immunotherapy: a challenge of breast cancer treatment. Cancers 2019; 11: 1822.

6. Basu A, Ramamoorthi G, Jia Y, et al. Immunotherapy in breast cancer: current status and future directions. Adv Cancer Res 2019; 143: 295-349.

7. Edechi CA, Ikeogu N, Uzonna JE, et al. Regulation of immunity in breast cancer. Cancers 2019; 11: 1080.

8. Keren L, Bosse M, Marquez D, et al. A structured tumor-immune microenvironment in triple negative breast cancer revealed by multiplexed ion beam imaging. Cell 2018; 174: 1373-87e19.

9. Clift R, Souratha J, Garrovillo SA, et al. Remodeling the tumor microenvironment sensitizes breast tumors to anti-programmed death-ligand 1 immunotherapy. Cancer Res 2019; 79: 4149-59.

10. Martinez-Outschoorn U, Sotgia F, Lisanti MP. Tumor microenvironment and metabolic synergy in breast cancers: critical importance of mitochondrial fuels and function. Semin Oncol 2014; 41: 195-216.

11. Canale FP, Ramello MC, Nunez N, et al. CD39 expression defines cell exhaustion in tumor-infiltrating CD8(+) T cells. Cancer Res 2018; 78: 115-28.

12. Gilbert SM, Oliphant CJ, Hassan S, et al. ATP in the tumour microenvironment drives expression of $\mathrm{nfP} 2 \mathrm{X} 7$, a key mediator of cancer cell survival. Oncogene 2019; 38: 194-208.

13. Park M, Kim J, Phuong NTT, et al. Involvement of the P2X7 receptor in the migration and metastasis of tamoxifen-resistant breast cancer: effects on small extracellular vesicles production. Sci Rep 2019; 9: 11587.

14. Hope JM, Greenlee JD, King MR. Mechanosensitive ion channels: TRPV4 and P2X7 in disseminating cancer cells. Cancer J 2018; 24: 84-92.

15. Adinolfi E, Giuliani AL, De Marchi E, et al. The P2X7 receptor: a main player in inflammation. Biochem Pharmacol 2018; 151: 234-44.

16. Draganov D, Gopalakrishna-Pillai S, Chen YR, et al. Modulation of P2X4/P2X7/Pannexin-1 sensitivity to extracellular ATP via Ivermectin induces a non-apoptotic and inflammatory form of cancer cell death. Sci Rep 2015; 5: 16222 .

17. Tan C, Han LI, Zou L, et al. Expression of P2X7R in breast cancer tissue and the induction of apoptosis by the gene-specific shRNA in MCF-7 cells. Exp Ther Med 2015; 10: 1472-8.

18. Huang S, Chen Y, Wu W, et al. miR-150 promotes human breast cancer growth and malignant behavior by targeting the pro-apoptotic purinergic $\mathrm{P} 2 \mathrm{X} 7$ receptor. PLoS One 2013; 8: e80707.

19. Thawkar BS, Kaur G. Inhibitors of NF-kappaB and P2X7/ NLRP3/Caspase 1 pathway in microglia: novel therapeutic opportunities in neuroinflammation induced early-stage Alzheimer's disease. J Neuroimmunol 2019; 326: 62-74.

20. Bae JY, Lee SW, Shin YH, et al. P2X7 receptor and NLRP3 inflammasome activation in head and neck cancer. Oncotarget 2017; 8: 48972-82.

21. Franceschini A, Capece M, Chiozzi P, et al. The P2X7 receptor directly interacts with the NLRP3 inflammasome scaffold protein. FASEB J 2015; 29: 2450-61.

22. Ghiringhelli F, Apetoh L, Tesniere A, et al. Activation of the NLRP3 inflammasome in dendritic cells induces 
IL-1 beta-dependent adaptive immunity against tumors. Nat Med 2009; 15: 1170-8.

23. Smeda M, Kieronska A, Proniewski B, et al. Dual antiplatelet therapy with clopidogrel and aspirin increases mortality in 4T1 metastatic breast cancer-bearing mice by inducing vascular mimicry in primary tumour. Oncotarget 2018; 9: 17810-24.

24. Wen Z, Mei B, Li H, et al. P2X7 participates in intracerebral hemorrhage-induced secondary brain injury in rats via MAPKs signaling pathways. Neurochem Res 2017; 42: 2372-83.

25. Qi Y, Hu X, Cui J, et al. Combined use of insoluble beta-glucan from the cell wall of Candida albicans and cyclophosphamide: Validation in S180 tumor-bearing mice. Biomed Pharmacother 2018; 97: 1366-72.

26. Im K, Mareninov S, Diaz MFP, et al. An introduction to performing immunofluorescence staining. Methods Mol Biol 2019; 1897: 299-311.

27. Burnstock G. Purinergic signalling: therapeutic developments. Front Pharmacol 2017; 8: 661.

28. Burnstock G, Knight GE. The potential of P2X7 receptors as a therapeutic target, including inflammation and tumour progression. Purinergic Signal 2018; 14: 1-18.

29. De Marchi E, Orioli E, Dal Ben D, et al. P2X7 receptor as a therapeutic target. Adv Protein Chem Struct Biol 2016; 104: 39-79.

30. Scarpellino G, Genova T, Munaron L. Purinergic P2X7 receptor: a cation channel sensitive to tumor microenvironment. Recent Pat Anticancer Drug Discov 2019; 14: 32-8.

31. Adinolfi E, De Marchi E, Orioli E, et al. Role of the P2X7 receptor in tumor-associated inflammation. Curr Opin Pharmacol 2019; 47: 59-64.

32. Arnaud-Sampaio VF, Rabelo ILA, Ulrich H, et al. The P2X7 receptor in the maintenance of cancer stem cells, chemoresistance and metastasis. Stem Cell Rev Rep 2020; 16: 288-300

33. Di Virgilio F, Giuliani AL, Vultaggio-Poma V, et al. Non-nucleotide agonists triggering $\mathrm{P} 2 \mathrm{X} 7$ receptor activation and pore formation. Front Pharmacol 2018; 9: 39.

34. Slater M, Danieletto S, Pooley M, et al. Differentiation between cancerous and normal hyperplastic lobules in breast lesions. Breast Cancer Res Treat 2004; 83: 1-10.

35. Salvestrini V, Orecchioni S, Talarico G, et al. Extracellular ATP induces apoptosis through P2X7R activation in acute myeloid leukemia cells but not in normal hematopoietic stem cells. Oncotarget 2017; 8: 5895-908.

36. Jelassi B, Anchelin M, Chamouton J, et al. Anthraquinone emodin inhibits human cancer cell invasiveness by antagonizing P2X7 receptors. Carcinogenesis 2013; 34: 1487-96.

37. McKelvey KJ, Hudson AL, Back M, et al. Radiation, inflammation and the immune response in cancer. Mamm Genome 2018; 29: 843-65.

38. Karki R, Man SM, Kanneganti TD. Inflammasomes and Cancer. Cancer Immunol Res 2017; 5: 94-9.

39. Yao M, Fan X, Yuan B, et al. Berberine inhibits NLRP3 Inflammasome pathway in human triple-negative breast cancer MDA-MB-231 cell. BMC Complement Altern Med 2019; 19: 216.

40. Raut PK, Kim SH, Choi DY, et al. Growth of breast cancer cells by leptin is mediated via activation of the inflammasome: critical roles of estrogen receptor signaling and reactive oxygen species production. Biochem Pharmacol 2019; 161: 73-88.

41. Biber K, Bhattacharya A, Campbell BM, et al. Microglial drug targets in AD: opportunities and challenges in drug discovery and development. Front Pharmacol 2019; 10: 840. 